\title{
Candiduria in hospitalized patients: an investigation with the Sysmex UF-1000i urine analyzer
}

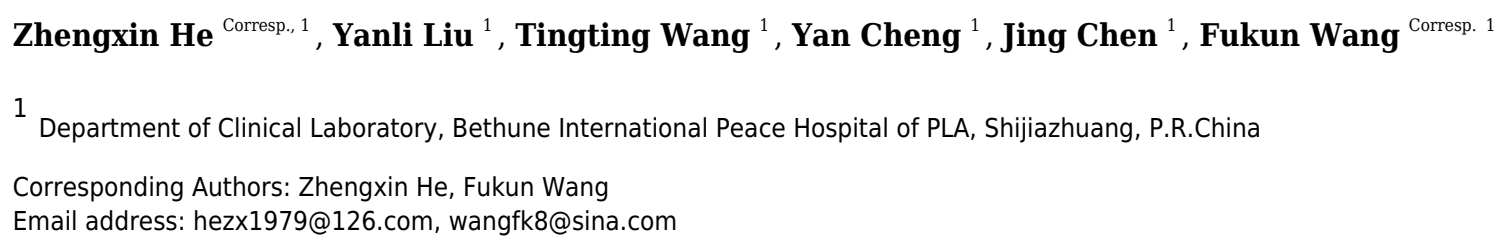

Background. Candiduria is common in hospitalized patients. Its management is limited because of inadequate understanding. Previous epidemiological studies based on culture assay have been limited to small study populations. Therefore, data collected by automated systems from a large target population are necessary for more comprehensive understanding of candiduria in hospitalized patients. Methods. To determine the performance of the Sysmex UF-1000i in detecting candiduria, a cross-sectional study was designed and conducted. A total of 203 yeast-like cell (YLC)-positive and 127 negative samples were randomly chosen and subjected to microbiologic analysis. The receiver operating characteristic curve (ROC) was used to evaluate the ability of YLC counts as measured by the Sysmex UF1000i to predict candiduria. Urinalysis data from 31,648 hospitalized patients were retrospectively investigated, and statistical analysis was applied to the data collected. Results. Using a cutoff value of $84.6 \mathrm{YLCs} / \mu \mathrm{L}$, the sensitivity, specificity, positive predictive value (PPV) and negative predictive value (NPV) of the yeast like cell (YLC) counts to predict candiduria were $61.7 \%, 84.1 \%, 88.6 \%$ and $66.3 \%$, respectively. C. glabrata (33.6\%) and C. tropicalis (31.4\%) were more prevalent than $C$. albicans $(24.3 \%)$ in the present study. Of the investigated hospitalized patients, 509 (1.61\%) were considered candiduria-positive. Age, gender and basic condition were associated with candiduria in hospitalized patients. In the ICU setting, urinary catheterization appeared to be the only independent risk factor contributing to candiduria according to our investigation. Although antibiotic therapy has been reported to be a very important risk factor, we could not confirm its significance in ICU candiduria patients because of excessive antibiotic usage in our hospital. Conclusions. The YLC measured by Sysmex UF-1000i is a practical and convenient tool for clinical candiduria screening prior to microbiologic culture. Candiduria is common in hospitalized patients, and its incidence varies according to age, gender and the wards where it is isolated. Candiduria had no direct connection with mortality but might be considered a marker of seriously ill patients who need particular attention in the clinic. 
1 Candiduria in hospitalized patients: an investigation with the Sysmex UF-1000i

2 urine analyzer

3

4 Authors:

5 Zhengxin He, Yanli Liu, Tingting Wang, Yan Cheng, Jing Chen and Fukun Wang*

6

7

8 Affiliations:

9 Department of Clinical Laboratory, Bethune International Peace Hospital of PLA, Shijiazhuang 10 050082, P.R.China;

11

12 Location:

13 Shijiazhuang, Hebei Province

14

15 Country:

16 China

17

18 Corresponding Author:

19 Professor Wang Fukun: Department of Clinical Laboratory, Bethune International Peace Hospital

20 of PLA, 398 Zhongshan Road, Shijiazhuang, Hebei 050082, P.R. China

21 E-mail: wangfk8@sina.com 


\section{Abstract}

23 Background. Candiduria is common in hospitalized patients. Its management is limited because

of inadequate understanding. Previous epidemiological studies based on culture assay have been limited to small study populations. Therefore, data collected by automated systems from a large target population are necessary for more comprehensive understanding of candiduria in hospitalized patients.

Methods. To determine the performance of the Sysmex UF-1000i in detecting candiduria, a crosssectional study was designed and conducted. A total of 203 yeast-like cell (YLC)-positive and 127 negative samples were randomly chosen and subjected to microbiologic analysis. The receiver operating characteristic curve (ROC) was used to evaluate the ability of YLC counts as measured by the Sysmex UF1000i to predict candiduria. Urinalysis data from 31,648 hospitalized patients were retrospectively investigated, and statistical analysis was applied to the data collected.

Results. Using a cutoff value of $84.6 \mathrm{YLCs} / \mu \mathrm{L}$, the sensitivity, specificity, positive predictive value (PPV) and negative predictive value (NPV) of the yeast like cell (YLC) counts to predict candiduria were $61.7 \%, 84.1 \%, 88.6 \%$ and $66.3 \%$, respectively. C. glabrata $(33.6 \%)$ and $C$. tropicalis $(31.4 \%)$ were more prevalent than C. albicans $(24.3 \%)$ in the present study. Of the investigated hospitalized patients, 509 (1.61\%) were considered candiduria-positive. Age, gender and basic condition were associated with candiduria in hospitalized patients. In the ICU setting, urinary catheterization appeared to be the only independent risk factor contributing to candiduria according to our investigation. Although antibiotic therapy has been reported to be a very important risk factor, we could not confirm its significance in ICU candiduria patients because of excessive antibiotic usage in our hospital.

Conclusions. The YLC measured by Sysmex UF-1000i is a practical and convenient tool for clinical candiduria screening prior to microbiologic culture. Candiduria is common in hospitalized patients, and its incidence varies according to age, gender and the wards where it is isolated. 
47 Candiduria had no direct connection with mortality but might be considered a marker of seriously 48 ill patients who need particular attention in the clinic.

49

50 
51

52

53

54

\section{Introduction}

2 Candiduria, defined as various Candida species found in the urine, is becoming an increasingly serious problem in hospitalized patients. Antibiotics, corticosteroids, immunosuppressive agents, and urinary catheters, all of which are used extensively in clinical practice, are regarded as possible risk factors [1]. The presence of Candida in urine does not always signify a fungal urinary tract infection (UTI) and may derive from colonization or sample contamination [2]. Nevertheless, candiduria could be considered a valuable clue to the diagnosis and treatment of fungal UTIs, especially in high-risk hospitalized patients. Numerous studies in experimental animals and clinical observations have indicated that the kidney consistently acts as the main fungus-bearing organ during candida infection [3-5]. In severely ill patients, candiduria has been considered to be significantly associated with disseminated candidiasis [6-8]. In surgical intensive care unit (ICU) settings, candiduria has been proved to be an independent factor associated with mortality [9].

For most clinical laboratories, urine culture is the first-line technique to determine candiduria. This conventional manual assay is time consuming, labor intensive and unsuitable for large-scale screening and investigation purposes. For a long time, flow cytometry was regarded as a useful tool for yeast characterization [10]. By utilizing fluorescent staining and flow cytometric technology, the Sysmex UF-1000i system measures each detected formed element, including fungi in urine, simply and efficiently. Several studies [11-13] have supported the reliability of an automatic urine analysis system for bacteria and yeast-like cell (YLC) detection.

Previous epidemiological studies of candiduria [14-16] have been based on culture assay and limited to small study populations. Hence, further data collected by automated systems from a large target population are necessary for more comprehensive understanding of candiduria in hospitalized patients. The aim of this study was to collect epidemiological data on candiduria with the Sysmex UF-1000i urine flow cytometry analysis system and to assess the incidence of candiduria in hospitalized patients. The risk factors and outcomes of ICU candiduria patients were 
76 also investigated in this study.

77

78

79

80

81

82

83

84

85

86

87

88

89

90

91

92

93

94

95

96

97

98

99

100

\section{Materials \& Methods}

\section{Study population}

This study was conducted between September 1, 2016, and August 31, 2017, in Bethune International Peace Hospital of PLA and was approved by the local research ethics committee (approval number: 2016-KY-034). We studied 45,371 urine samples collected from 31,648 hospitalized patients, including 17,840 males and 13,808 females. The enrolled patients were aged 1 day to 106 years old with a median age of 56 years. Samples from infants younger than 3 years accounted for $8.86 \%$ of the total number of samples. Clinical data, including age, gender, primary diagnosis, risk factors (e.g., presence of catheter, antibiotics, parenteral nutrition, immunosuppressive treatment, renal failure, abdominal surgery, diabetes and malignancy) and prognosis, were obtained from medical records. For ICU patients, Acute Physiology and Chronic Health Evaluation (APACHE) II scores were used to evaluate ICU admission severity.

Patients were instructed on how to collect mid-stream urine before sample collection. For babies younger than 1 year, a sterile urine collection bag was applied according to the manufacturer's instructions. The samples were collected in the morning and were stored in a disposable sterile bottle with a screw lid. All samples were analyzed within 3 hours after collection.

All urine samples received were examined with a Sysmex UF-1000i analysis system and the count of yeast-like cells (YLCs) was used as a candiduria marker [11]. For patients who underwent repeated urine examinations, the maximum YLC count value was recorded.

\section{Microbiology analysis}

To determine the performance of the Sysmex UF-1000i in detecting candiduria, a cross-sectional study was designed and conducted. A total of 203 YLC-positive and 127 negative samples were randomly chosen and subjected to microbiological analysis. Briefly, $0.01 \mathrm{ml}$ of each urine sample 
101

102

103

104

105

106

107

108

109

110

111

112

113

114

115

116

117

118

119

120

121

122

123

124

125

was spread on a CHROMagar plate with a calibrated loop, and the culture plates were incubated aerobically at $35^{\circ} \mathrm{C}$ for 48 hours. The Candida species were identified based on growth color and colony shape and were further confirmed by the API 20C AUX Yeast Identification System (BioMerieux, France). The determined level for quantitative cultures to be considered significant was $10^{3} \mathrm{CFU} / \mathrm{ml}(10 \mathrm{CFU} /$ plate $)$.

\section{Statistical analysis}

The LOG YLC counts results of the culture-positive/negative groups are given as mean \pm standard deviation (SD). The candiduria rate according to age groups, genders and wards were statistically compared using Fisher's exact test. Risk factors for ICU patients were assessing by logistic regression. Statistical analysis of YLC counts were performed with the Mann-Whitney test. A $P$ value $<0.05$ was considered significant. Statistical analysis was performed using software GraphPad Prism 7.0 and SPSS 17.0. The characteristics of YLC for determining candiduria, including sensitivity, specificity, positive predictive value (PPV), and negative predictive value (NPV), were calculated.

\section{Results}

\section{YLC detection capacity of the Sysmex UF1000i and cutoff value determination}

By microbiological culture assay, we isolated 132 strains of Candida species from 119 (119/203) YLC-positive samples and 8 strains from 8 (8/127) YLC-negative samples. Table 1 displays the prevalence of the 140 isolated Candida species strains, including 47 strains of Candida glabrata, 44 strains of Candida tropicalis, 34 strains of Candida albicans, 6 strains of Candida krusei, 4 strains of Candida parapsilosis and 5 strains of other species.

Figure 1A shows the YLC counts obtained from the YLC-positive samples. Compared to that of the culture-negative group, the YLC count (LOG n) of the culture-positive group was significantly higher $(2.259 \pm 0.067 \mathrm{VS} 1.524 \pm 0.052, P<0.001)$. The receiver operating characteristic curve 
126 (ROC) was utilized to evaluate the predictive ability of the Sysmex UF1000i YLC count to predict

127 candiduria. The sensitivity and specificity were calculated based on a determined cutoff value of

128 the ROC curve. As Figure 1B shows, the area under the curve (AUC) was 0.789 with a 95\% 129 confidence interval of $0.728-0.851$. The Youden index (sensitivity + specificity -1$)$ was 130 generated, and 84.6 YLCs/ $\mu \mathrm{L}$ was determined as the best cutoff value. The sensitivity, specificity, 131 PPV and NPV of the YLC counts to determine candiduria were $61.7 \%, 84.1 \%, 88.6 \%$ and $66.3 \%$, 132 respectively.

133 Characteristics of patients with candiduria

134 For the investigated samples, 684 of $45,371(1.51 \%)$ were determined to be positive, and 509 of 135 $31,648(1.61 \%)$ patients were considered candiduria-positive with a YLC cutoff value of 84.6

136

137

138 139

140

141

142

143

144

145

146

147

148

149

150

$\mathrm{YLCs} / \mu \mathrm{L}$.

Table 2 shows the age and gender distribution of the candiduria patients. The enrolled patients were divided into 5 age groups, and per-age maximum candiduria determination was displayed as follows: $<1$ group (3.41\%), followed by the $60+(2.51 \%)$ and $18 \sim 59$ groups $(1.06 \%)$. The $1 \sim 6$ $(0.20 \%)$ and $7 \sim 17$ groups $(0.54 \%)$ showed very low candiduria rates. Of the 509 positive patients, 235 were male, and 274 were female, and the candiduria occurrence rate was statistically higher in female patients than in male patients $(P<0.001)$.

Underlying disease was recognized as the main factor for candiduria. Table 3 exhibits the ward distribution of the candiduria patients. Generally, candiduria rates in patients from internal medicine wards were more than two times higher than those of patients in the surgical wards (1.98\% vs $0.94 \%, P<0.001)$. The ICU (22.89\%), nephrology $(7.06 \%)$, geriatrics $(5.66 \%)$, NICU $(3.66 \%)$, neurosurgery $(2.94 \%)$, hematology $(2.78 \%)$, oncology $(2.60 \%)$ and respiratory $(2.54 \%)$ wards were the leading wards for candiduria, with occurrence rates were significantly higher than the average rate $(1.61 \%)$.

Risk factors and outcome of ICU candiduria patients

Peer) reviewing PDF | (2018:09:31040:3:0:NEW 3 Apr 2019) 
151 Subjects in this study included 166 patients from the ICU of our hospital, among whom 38 were 152 identified as having candiduria (38/166, 22.89\%). Previous reports showed high candiduria rates 153 in ICU patients, and we further investigated the risk factors and outcomes of the ICU candiduria 154 patients.

155

156

157

158

159

160

161

162

163

164

165

166

167

168

169

170

171

172

173

174

175

Among the several risk factors investigated, the presence of a catheter was found to be statistically significant by multi-factor logistic regression analysis $(P<0.05$, Table 4$)$. Other factors, including age $>60$ years, antibiotic and immunosuppressive therapy, parenteral nutrition, abdominal surgery, diabetes, malignancy, renal failure and APACHE II scores were not found to be statistically significant.

A total of 26 ICU investigated patients died in hospital (13 were identified with candiduria and 13 did not). The death rate was significantly higher among candiduria patients than among those without candiduria (13/38 VS 13/128). For the 38 ICU candiduria subjects, 8 received antifungal therapy, and 2 of them died; 30 did not receive antifungal treatment, and 11 of them died. There was no significant difference between the death rates of patients who received antifungal therapy and of those who did not (2/8 VS 11/30). One candiduria patient was clinically diagnosed as having candidemia with a positive blood culture of Candida albicans. This patient died within 15 days despite aggressive antifungal therapy.

\section{Discussion}

The Sysmex UF-1000i is a fully automatic fluorescence cytometric urine analyzer capable of detecting various urine particles, including bacteria (BACT), red cells (RBCs), white cells (WBCs), yeast-like cells (YLCs), epithelial cells, casts, crystals, spermatozoa, small round cells and mucus [17]. Several studies [11-13] have shown that the Sysmex UF-1000i analyzer can determine UTIs and candiduria in a simple manner with considerable quantification. Our data indicated that the sensitivity, specificity, PPV, and NPV were $61.7 \%, 84.1 \%, 88.6 \%$ and $66.3 \%$, 
176

177

178

179

180

181

182

183

184

185

186

187

188

189

190

191

192

193

194

195

196

197

198

199

200

respectively, using a cutoff value of $84.6 \mathrm{YLCs} / \mu \mathrm{L}$. According to a report from GutiérrezFernández $\mathrm{J}$ et al.[11], the sensitivity, specificity, PPV and NPV of urine YLC counts for determining candiduria were $87.3 \%, 97.0 \%, 9.3 \%$ and $99.9 \%$, respectively, with a cutoff value of $\geq 50 \mathrm{YLCs} / \mu \mathrm{L}$. Compared to the present study, their study used a relatively low cutoff value and enrolled all YLC-negative subjects into the calculation, leading to a very low PPV. When determining the cutoff value, we only included the YLC-positive subjects in the calculation because the main question of the present study was whether positive YLC indicated candiduria. The urinary tract is one of the most frequent sites for candida colonization, and the presence of candida in urine should not be ignored because candida colonization is a risk factor for the progress of invasive candidiasis (IC) $[18,19]$. In our study, the general incidence of candiduria was $1.61 \%$ in hospitalized patients. Actually, according to limited data available, the candiduria incidence rates were varied from $0.77 \%$ to over $20 \%[15,20]$. Our result was comparable to a clinic-based retrospective and prospective study using culture assay conducted by Colodner et al.[20]. Compared to study data regarding candiduria epidemiology in community settings, by which the candiduria rates were reported respectively as low as $0.4 \%$ [11] and $0.14 \%$ [20], our investigation result is much higher in hospitalized patients. This result indicates that the incidence of candiduria varied according to subject groups. C. glabrata and C. tropicalis were found more often than was C. albicans, agreeing with the trend of non-albicans Candida prevalence worldwide. Especially in the urinary tract, non-albicans Candida species were reported better-adapted to the environment [21]. C. glabrata [22] and C. tropicalis [23] were identified as the most prevalent urinary tract Candida species in various studies.

Candiduria occurrence rates varied according to age groups. The incidence was $2.51 \%$ in patients older than 60 years and $3.41 \%$ in patients younger than 1 year, significantly higher than the overall incidence rate (1.61\%). Older age is a classical risk factor for candiduria[24]. In healthy babies, the urinary tract is generally considered to be clean and without candida colonization, while in 
201

202

203

204

205

206

207

208

209

210

211

212

213

214

215

216

217

218

219

220

221

222

223

224

225

NICU patients, particularly premature infants, candiduria and candidemia were not rare [25, 26]. Gender is another factor that may affect the candiduria incidence [27]. In the present study, the candiduria incidence was significantly higher in females than in males. A possible explanation to this finding may be the common colonization of Candida in the urogenital tract or Candida vulvovaginitis in females $[20,28]$.

To explore the correlation between underlying diseases and candiduria in hospitalized patients, the candiduria incidences in patients who were admitted to various wards were investigated. As expected, ICU patients showed the highest candiduria incidence (22.89\%). Patients from nephrology (7.06\%), geriatrics (5.66\%), NICU (3.66\%), neurosurgery (2.94\%), hematology $(2.78 \%)$ and oncology $(2.60 \%)$ wards also showed considerably high candiduria rates. This result might imply that stays in the ICU, major surgery, very old/young age, diabetes, renal disease, malignancy and immunosuppressive therapy, in addition to gender, could be considered to be major risk factors for candiduria. For patients undergoing neurosurgery, whether a urinary catheter was used in the peri-operative period is very important for the development of candiduria [28]. In the present study, all 274 patients undergoing head operations had a record of urinary catheter usage that might be a major cause of the high prevalence of candiduria in the neurosurgery ward. In ICU patients, urinary catheterization appeared to be statistically significant risk factor. The presence of a catheter in the urinary tract was always an important factor associated with candiduria and various UTIs, as confirmed by many studies [29-32]. Previous antibiotic therapy has been considered to be another important risk factor, playing a crucial role in the pathogenesis of candiduria [33]. Excessive antibiotic use is widely recognized in the ICU patients we investigated (142 of 166 patients had a record of antibiotic therapy). The high prevalence of antibiotic use in ICU patients makes it difficult to clarify the role of antibiotic therapy in the prevalence of candiduria or Candida colonization in our report.

The total mortality rate for ICU candiduria patients was $34.21 \%$, approximately 3 times higher 
226

227

228

229

230

231

232

233

234

235

236

237

238

239

240

241

242

243

244

245

246

247

248

249

250

than that of the non-candiduria patients. This figure is very close to the mortality rate reported by Bougnoux ME et al. [34]. Antifungal treatment had no effect on the mortality of ICU candiduria patients in our investigation. It appears likely that candiduria is a "red flag" or marker for seriously ill patients who need particular attention to their indwelling devices or underlying disease but has no direct contribution to the high mortality. Although the connection between candiduria and candidemia has been reported by several groups [35], only 1 ICU candiduria patient was finally diagnosed as having candidemia by microbiological culture in this study.

The findings of this study have to be seen in light of some limitations. First, the epidemiological data are obtained from the Sysmex UF-1000i YLC parameter. The performance of YLC for candiduria screening is relatively low, which might bias the results of our overall and subgroup analyses. However, retrospectively investigating candiduria with YLC could greatly reduce the selection bias caused by target population, for the reason that urine culture is warranted only when the patient exhibits urinary tract infection symptoms in clinical practice, while urinalysis is performed as a routine examination on each patient. Second, the sample size for ICU patients is not big enough. Third, as a retrospective study, we only conduct follow-up evaluation for the outcome of ICU patients.

\section{Conclusions}

To our knowledge, this is the first epidemiological study of candiduria with a large-scale investigated population conducted using the Sysmex UF-1000i urine analyzer. The incidence rate of candiduria in hospitalized patients was approximately $1.61 \%$ and varied according to age, gender and the wards where they were isolated. Presence of catheter was the only independent risk factor in ICU patients. Antifungal therapy had no effect on the mortality of ICU candiduria patients, implying that candiduria was not directly associated with mortality.

\section{Acknowledgments}

Peer) reviewing PDF | (2018:09:31040:3:0:NEW 3 Apr 2019) 
251 We thank all the physicians and nurses who have contributed to the research. 
252

25

254

255

256

257

258

259

260

261

262

263

264

265

266

267

268

269

270

271

272

273

274

275

276

277

278

279

280

281

282

283

284

285

286

287

288

289

290

291

292
1. Fakhri A, Navid M, Seifi Z, Zarei Mahmoudabadi A. The frequency of candiduria in hospitalized patients with depressive syndrome. Journal of Renal Injury Prevention. 2014; 34:97-8.

2. Kauffman CA, Fisher JF, Sobel JD, Newman CA. Candida urinary tract infections-diagnosis. Clinical infectious diseases : an official publication of the Infectious Diseases Society of America. 2011; 52 Suppl 6:S452-6.

3. Chowdhury MH, Ryan LK, Cherabuddi K. Antifungal Potential of Host Defense Peptide Mimetics in a Mouse Model of Disseminated Candidiasis. 2018; 41.

4. Spellberg B, Ibrahim AS, Edwards JE, Jr., Filler SG. Mice with disseminated candidiasis die of progressive sepsis. The Journal of infectious diseases. 2005; 1922:336-43.

5. Wang X, Fries BC. A murine model for catheter-associated candiduria. Journal of medical microbiology. 2011; 60Pt 10:1523-9.

6. Drogari-Apiranthitou M, Anyfantis I, Galani I, Kanioura L, Daikos GL, Petrikkos G. Association Between Candiduria and Candidemia: A Clinical and Molecular Analysis of Cases. Mycopathologia. 2017.

7. Huang PY, Hung MH, Shie SS, Su LH, Chen KY, Ye JJ, Chiang PC, Leu HS, Huang CT. Molecular concordance of concurrent Candida albicans candidemia and candiduria. Diagnostic microbiology and infectious disease. 2013; 763:382-4.

8. Bougnoux ME, Kac G, Aegerter P, d'Enfert C, Fagon JY. Candidemia and candiduria in critically ill patients admitted to intensive care units in France: incidence, molecular diversity, management and outcome. Intensive care medicine. 2008; 342:292-9.

9. Magill SS, Swoboda SM, Johnson EA, Merz WG, Pelz RK, Lipsett PA, Hendrix CW. The association between anatomic site of Candida colonization, invasive candidiasis, and mortality in critically ill surgical patients. Diagnostic microbiology and infectious disease. 2006; 554:293-301.

10. Huls PG, Nanninga N, van Spronsen EA, Valkenburg JA, Vishcer NO, Woldringh CL. A computer-aided measuring system for the characterization of yeast populations combining 2D-image analysis, electronic particle counter, and flow cytometry. Biotechnology and bioengineering. 1992; 393:343-50.

11. Gutierrez-Fernandez J, Riazzo C, Sanbonmatsu S, de Dios Luna J, Sorlozano A, Miranda C, Navarro JM. Sysmex UF-1000i performance for screening yeasts in urine. APMIS : acta pathologica, microbiologica, et immunologica Scandinavica. 2014; 1224:324-8.

12. Le Z, Li F, Fei C, Ye A, Xie X, Zhang J. Performance of the Sysmex UF-1000i urine analyser in the rapid diagnosis of urinary tract infections in hospitalized patients. Journal of infection and chemotherapy : official journal of the Japan Society of Chemotherapy. 2016; 226:377-82.

13. Manoni F, Fornasiero L, Ercolin M, Tinello A, Ferrian M, Hoffer P, Valverde S, Gessoni G. Cutoff values for bacteria and leukocytes for urine flow cytometer Sysmex UF-1000i in urinary tract infections. Diagnostic microbiology and infectious disease. 2009; 652:10307. 
293

294

295

296

297

298

299

300

301

302

303

304

305

306

307

308

309

310

311

312

313

314

315

316

317

318

319

320

321

322

323

324

325

326

327

328

329

330

331

332

333

14. Aubron C, Suzuki S, Glassford NJ, Garcia-Alvarez M, Howden BP, Bellomo R. The epidemiology of bacteriuria and candiduria in critically ill patients. Epidemiology and infection. 2015; 1433:653-62.

15. Kobayashi CC, de Fernandes OF, Miranda KC, de Sousa ED, Silva Mdo R. Candiduria in hospital patients: a study prospective. Mycopathologia. 2004; 1581:49-52.

16. Paul N, Mathai E, Abraham OC, Michael JS, Mathai D. Factors associated with candiduria and related mortality. The Journal of infection. 2007; 555:450-5.

17. De Rosa R, Grosso S, Bruschetta G, Avolio M, Stano P, Modolo ML, Camporese A. Evaluation of the Sysmex UF1000i flow cytometer for ruling out bacterial urinary tract infection. Clinica chimica acta; international journal of clinical chemistry. 2010; 4111516:1137-42.

18. Lau AF, Kabir M, Chen SC, Playford EG, Marriott DJ, Jones M, Lipman J, McBryde E, Gottlieb T, Cheung W, Seppelt I, Iredell J, Sorrell TC. Candida colonization as a risk marker for invasive candidiasis in mixed medical-surgical intensive care units: development and evaluation of a simple, standard protocol. Journal of clinical microbiology. 2015; 534:1324-30.

19. Delaloye J, Calandra T. Invasive candidiasis as a cause of sepsis in the critically ill patient. Virulence. 2014; 51:161-9.

20. Colodner R, Nuri Y, Chazan B, Raz R. Community-acquired and hospital-acquired candiduria: comparison of prevalence and clinical characteristics. European journal of clinical microbiology \& infectious diseases : official publication of the European Society of Clinical Microbiology. 2008; 274:301-5.

21. Sobel JD, Fisher JF, Kauffman CA, Newman CA. Candida urinary tract infections-epidemiology. Clinical infectious diseases : an official publication of the Infectious Diseases Society of America. 2011; 52 Suppl 6:S433-6.

22. Safdar N, Slattery WR, Knasinski V, Gangnon RE, Li Z, Pirsch JD, Andes D. Predictors and outcomes of candiduria in renal transplant recipients. Clinical infectious diseases : an official publication of the Infectious Diseases Society of America. 2005; 4010:1413-21.

23. Singla N, Gulati N, Kaistha N, Chander J. Candida colonization in urine samples of ICU patients: determination of etiology, antifungal susceptibility testing and evaluation of associated risk factors. Mycopathologia. 2012; 1742:149-55.

24. Fraisse T, Crouzet J, Lachaud L, Durand A, Charachon S, Lavigne JP, Sotto A. Candiduria in Those over 85 Years Old: A Retrospective Study of 73 Patients. Internal Medicine. 2011; 5018:1935-40.

25. Kelly MS, Benjamin DK, Jr, Smith PB. The epidemiology and diagnosis of invasive candidiasis among premature infants. Clinics in perinatology. 2015; 421:105-17, viii-ix.

26. Swanson JR, Gurka MJ, Kaufman DA. Risk Factors for Invasive Fungal Infection in Premature Infants: Enhancing a Targeted Prevention Approach. Journal of the Pediatric Infectious Diseases Society. 2014; 31:49-56.

27. Kauffman CA, Vazquez JA, Sobel JD, Gallis HA, McKinsey DS, Karchmer AW, Sugar AM, Sharkey PK, Wise GJ, Mangi R, Mosher A, Lee JY, Dismukes WE. Prospective 
334

335

336

337

338

339

340

341

342

343

344

345

346

347

348

349

350

351

352

353

354

355

356

357

358

359

360

361

362

multicenter surveillance study of funguria in hospitalized patients. The National Institute for Allergy and Infectious Diseases (NIAID) Mycoses Study Group. Clinical infectious diseases : an official publication of the Infectious Diseases Society of America. 2000; 301:14-8.

28. Ksycki MF, Namias N. Nosocomial urinary tract infection. The Surgical clinics of North America. 2009; 892:475-81, ix-X.

29. Padawer D, Pastukh N, Nitzan O, Labay K, Aharon I, Brodsky D, Glyatman T, Peretz A. Catheter-associated candiduria: Risk factors, medical interventions, and antifungal susceptibility. American journal of infection control. 2015; 437:e19-22.

30. Nett JE, Brooks EG, Cabezas-Olcoz J, Sanchez H, Zarnowski R, Marchillo K, Andes DR. Rat indwelling urinary catheter model of Candida albicans biofilm infection. Infection and immunity. 2014; 8212:4931-40.

31. Sobel JD, Kauffman CA, McKinsey D, Zervos M, Vazquez JA, Karchmer AW, Lee J, Thomas C, Panzer H, Dismukes WE. Candiduria: a randomized, double-blind study of treatment with fluconazole and placebo. The National Institute of Allergy and Infectious Diseases (NIAID) Mycoses Study Group. Clinical infectious diseases : an official publication of the Infectious Diseases Society of America. 2000; 301:19-24.

32. Kim B, Pai H, Choi WS, Kim Y, Kweon KT, Kim HA, Ryu SY, Wie SH, Kim J. Current status of indwelling urinary catheter utilization and catheter-associated urinary tract infection throughout hospital wards in Korea: A multicenter prospective observational study. 2017; 1210:e0185369.

33. Weinberger M, Sweet S, Leibovici L, Pitlik SD, Samra Z. Correlation between candiduria and departmental antibiotic use. The Journal of hospital infection. 2003; 533:183-6.

34. Bougnoux ME, Kac G, Aegerter P, d'Enfert C, Fagon JY, CandiRea Study G. Candidemia and candiduria in critically ill patients admitted to intensive care units in France: incidence, molecular diversity, management and outcome. Intensive care medicine. 2008; 342:292-9.

35. Pemán J, Ruiz-Gaitán A. Candidemia from urinary tract source: the challenge of candiduria. Hospital Practice. 2018; DOI: 10.1080/21548331.2018.1538623. 
Figure 1

Figure 1 Yeastlike cells (YLC) performance for screening candiduria.

(A) For YLC positive samples, YLC count (LOG $n$ ) of culture positive samples is significantly higher than culture negative samples $(P<0.001)$. The error bars represent the standard deviations; (B) The receiver operating characteristic curve (ROC) was utilized to determine the cutoff YLC value for candiduria identifying. The area under the curve (AUC) was 0.789 and the cutoff value was determined as 84.6 YLCs $/ \mu \mathrm{L}$.
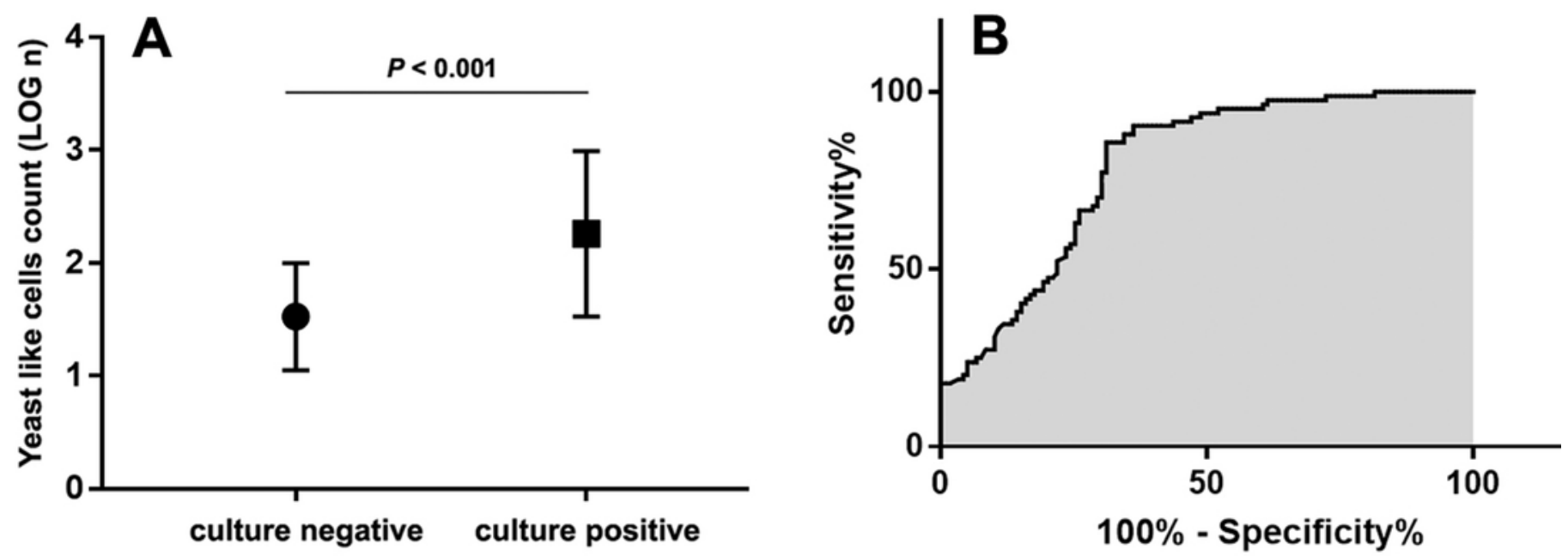


\section{Table 1 (on next page)}

Prevalence of candida species in the urine investigated by culture

${ }^{a} 13$ patients had $>1$ candida species isolated at baseline.

${ }^{\mathrm{b}}$ Includes Candida guilliermondii(2), Candida Iusitaniae (1), Saccharomyces cerevisiae (1) and Candida pseudotropicalis (1). 
Table 1 prevalence of candida species in the urine investigated by culture

\begin{tabular}{|c|c|}
\hline Candida species & No. $(\%)$ indentified ${ }^{a}$ \\
\hline C. glabrata & $47(33.6)$ \\
\hline C. tropicalis & $44(31.4)$ \\
\hline C. albicans & $34(24.3)$ \\
\hline C. krusei & $6(4.29)$ \\
\hline C. parapsilosis & $4(2.86)$ \\
\hline Other $C . s p p .{ }^{\mathrm{b}}$ & $5(3.57)$ \\
\hline
\end{tabular}

2 a 13 patients had $>1$ candida species isolated at baseline.

3 b Includes Candida guilliermondii(2), Candida lusitaniae (1), Saccharomyces cerevisiae (1) and Candida 4 pseudotropicalis(1).

5 
Table 2 (on next page)

Age and gender of 509 patients with candiduria 
1 Table 2 Age and gender of 509 patients with candiduria

\begin{tabular}{lcc}
\hline \multicolumn{1}{c}{ Characteristic } & $\begin{array}{c}\text { Candiduria } \\
(\mathrm{n}=509)\end{array}$ & $\begin{array}{c}\text { Non-candiduria } \\
(\mathrm{n}=31,139)\end{array}$ \\
\hline Age, years, $\mathrm{n}(\%)$ & & \\
$<1$ & $49(3.41)$ & 1,387 \\
$\quad 1 \sim 6$ & $5(0.20)$ & 2,553 \\
$\quad 7 \sim 17$ & $7(0.54)$ & 1,292 \\
$\quad 18 \sim 59$ & $157(1.06)$ & 14,604 \\
$\quad 60 \sim$ & $291(2.51)$ & 11,303 \\
Gender & & 17,605 \\
Male & $235(1.32)$ & 13,534 \\
Female & $274(1.98)$ & \\
\hline
\end{tabular}

2 


\section{Table 3(on next page)}

Number of candiduria patients from different medical wards

${ }^{a}$ CCU: cardiac care unit; NICU: neonatal intensive care unit; ICU: intensive care unit; ENT: ear, nose and throat.

${ }^{\mathrm{b}}$ Compared with overall candiduria incidence rate (509/31648, 1.61\%); $P$ value $<0.05$ was considered significant. 
1 Table 3 Number of candiduria patients from different medical wards

\begin{tabular}{|c|c|c|c|}
\hline Ward $^{\mathrm{a}}$ & Candiduria, \% & Non-candiduria & $P$ value ${ }^{\mathrm{b}}$ \\
\hline Internal medicine & $411(1.98)$ & 20,802 & $0.02^{*}$ \\
\hline $\mathrm{CCU}$ & $18(1.38)$ & 1,284 & 0.57 \\
\hline Gynaecology & $10(0.60)$ & 1,654 & $0.0015^{*}$ \\
\hline Infectious disease & $9(1.74)$ & 508 & 0.78 \\
\hline Hepatology & $5(0.62)$ & 800 & $0.03^{*}$ \\
\hline Pediatrics & $9(0.33)$ & 2,729 & $<0.0001^{*}$ \\
\hline NICU & $42(3.66)$ & 1,104 & $<0.0001^{*}$ \\
\hline Respiratory & $47(2.54)$ & 1,804 & $0.0016^{*}$ \\
\hline Emergency & $2(2.22)$ & 88 & 0.66 \\
\hline Geriatrics & $65(5.66)$ & 1,083 & $<0.0001^{*}$ \\
\hline Endocrinology & $19(1.58)$ & 1,180 & 1.00 \\
\hline Neurology & $12(0.72)$ & 1,656 & $0.0052^{*}$ \\
\hline Nephrology & $68(7.06)$ & 895 & $<0.0001^{*}$ \\
\hline Gastroenterology & $22(1.68)$ & 1,291 & 0.79 \\
\hline Cardiovascular & $7(0.30)$ & 2,303 & $<0.0001^{*}$ \\
\hline Hematology & $11(2.78)$ & 384 & 0.06 \\
\hline Rheumatology & $8(1.22)$ & 649 & 0.46 \\
\hline Oncology & $19(2.60)$ & 712 & $0.03^{*}$ \\
\hline ICU & $38(22.89)$ & 128 & $<0.0001^{*}$ \\
\hline Surgery wards & $98(0.94)$ & 10,337 & $<0.0001^{*}$ \\
\hline Hepatobiliary & $4(0.65)$ & 615 & 0.07 \\
\hline ENT & $11(0.39)$ & 2,793 & $<0.0001^{*}$ \\
\hline Orthopaedics & $13(0.84)$ & 1,530 & $0.02^{*}$ \\
\hline Urology & $17(1.74)$ & 961 & 0.70 \\
\hline General surgery & $16(0.87)$ & 1,833 & $0.015^{*}$ \\
\hline Neurosurgery & $19(2.94)$ & 627 & $0.0066^{*}$ \\
\hline Vascular surgery & 7 (1.09) & 637 & 0.32 \\
\hline Cardiothoracic & $1(0.26)$ & 391 & $0.04^{*}$ \\
\hline Ophthalmology & $4(0.55)$ & 726 & $0.02^{*}$ \\
\hline Obstetrics & $6(0.77)$ & 774 & 0.07 \\
\hline
\end{tabular}

2 a CCU: cardiac care unit; NICU: neonatal intensive care unit; ICU: intensive care unit; ENT: ear, nose and 3 throat.

4 b Compared with overall candiduria incidence rate $(509 / 31648,1.61 \%) ; P$ value $<0.05$ was considered 5 significant.

$6 \quad{ }^{*} P<0.05$. 


\section{Table 4(on next page)}

Table 4 Risk factors and outcome for ICU candiduria patients

${ }^{*}, P$ value $<0.05$ 


\begin{tabular}{|c|c|c|c|c|c|c|}
\hline \multirow{2}{*}{ Characteristic for ICU patients } & \multirow{2}{*}{$\begin{array}{l}\text { Candiduria (n, } \\
\%)\end{array}$} & \multirow{2}{*}{$\begin{array}{c}\text { Non-candiduria }(\mathrm{n}, \\
\%)\end{array}$} & \multicolumn{2}{|c|}{ Single-factor logistic regression analysis } & \multicolumn{2}{|c|}{ Multi-factor logistic regression analysis } \\
\hline & & & $P$ value & Odds ratio $(95 \% \mathrm{CI})$ & $P$ value & Odds ratio $(95 \% \mathrm{CI})$ \\
\hline \multicolumn{7}{|l|}{ Risk factors } \\
\hline $\begin{array}{l}\text { APACHE II score (median, } \\
\text { interquartile range) }\end{array}$ & $17(12-23)$ & $13(9-16)$ & $0.001^{*}$ & $1.109(1.047-1.175)$ & 0.774 & $0.932(0.576-1.507)$ \\
\hline$>60$ years & $28(73.68)$ & $90(70.31)$ & 0.561 & $1.273(0.564-2.870)$ & 0.911 & $1.051(0.437-2.532)$ \\
\hline Presence of catheter & $22(57.89)$ & $41(32.03)$ & $0.004^{*}$ & $3.025(1.437-6.369)$ & $0.006^{*}$ & $3.161(1.385-7.212)$ \\
\hline Antibiotics & $33(86.84)$ & $109(85.16)$ & 0.795 & $1.150(0.399-3.318)$ & 0.633 & $0.749(0.228-2.456)$ \\
\hline Parenteral nutrition & $23(60.53)$ & $69(53.91)$ & 0.665 & $1.176(0.566-2.444)$ & 0.965 & $0.981(0.417-2.308)$ \\
\hline Immunosuppressive & $2(5.26)$ & $3(2.34)$ & 0.368 & $2.315(0.372-14.390)$ & 0.883 & $1.192(0.115-12.377)$ \\
\hline \multicolumn{7}{|l|}{ treatment } \\
\hline Abdominal surgery & $4(10.52)$ & $7(5.47)$ & 0.377 & $1.765(0.501-6.216)$ & 0.366 & $1.902(0.472-7.664)$ \\
\hline Renal failure & $19(50.0)$ & $38(29.69)$ & 0.057 & $2.054(0.980-4.303)$ & 0.077 & $2.203(0.917-5.291)$ \\
\hline Diabetes & $14(36.84)$ & $35(27.34)$ & 0.262 & $1.550(0.721-3.332)$ & 0.473 & $1.372(0.579-3.251)$ \\
\hline Malignancy & $6(15.79)$ & $12(9.38)$ & 0.269 & $1.812(0.631-5.207)$ & 0.098 & $3.348(0.801-13.996)$ \\
\hline \multicolumn{7}{|l|}{ Outcome } \\
\hline Dead in hospital & $13(34.21)$ & $13(10.16)$ & $0.001^{*}$ & $4.600(1.904-11.113)$ & - & - \\
\hline Discharge & $25(65.79)$ & $115(89.84)$ & - & - & - & - \\
\hline
\end{tabular}

${ }^{*}, P$ value $<0.05$. 